\title{
INTERACTIVE TRUNK EXTRACTION FROM FOREST POINT CLOUD
}

\author{
T. Mizoguchi a ${ }^{\text {, Y. Kobayashi }}{ }^{\text {a }}$
}

${ }^{a}$ Department of Computer Science, College of Engineering, Nihon University

1 Nakagawara, Tokusada, Tamuramachi, Koriyama, Fukushima, 963-8642, Japan

(mizo, koba)@cs.ce.nihon-u.ac.jp

\section{Commission V}

KEY WORDS: Terrestrial Laser Scanning, Forest, User-Interaction

\begin{abstract}
:
For forest management or monitoring, it is required to constantly measure several parameters of each tree, such as height, diameter at breast height, and trunk volume. Terrestrial laser scanner has been used for this purpose instead of human workers to reduce time and cost for the measurement. In order to use point cloud captured by terrestrial laser scanner in the above applications, it is an important step to extract all trees or their trunks separately. For this purpose, we propose an interactive system in which a user can intuitively and efficiently extract each trunk by a simple editing on the distance image created from the point cloud. We demonstrate the effectiveness of our proposed system from various experiments.
\end{abstract}

\section{INTRODUCTION}

Recently terrestrial laser scanner has gaining much attention as its performance improves and been widely used in many applications, such as water resource investigation (Molina, 2014), as-built modelling of pipelines (Kawashima, 2014 and Lee, 2013), and damage evaluation of concrete structures (Mizoguchi, 2013). The main advantage of the laser scanner is efficient and detailed documentation of the concerned targets. This scanner is also well utilized in forestry community to capture the detailed and reliable information of individual tree without time-consuming labours by human workers. Therefore many advanced studies have been reported based on laser scanning of forest, e.g., approximation of crown volume and branch size distribution (Raumonen, 2011), leaf area density evaluation (Hosoi, 2006), leaf orientation retrieval (Zheng, 2012), stem curve measurement (Liang, 2014), and aboveground biomass estimation (Kankare, 2013 and Yu, 2013).

\subsection{Related works}

In order to use point cloud of forest captured by terrestrial laser scanner in the above applications, it is a crucial step to extract all of the trees or their trunks on scans separately. For this purpose, many automatic methods have been reported.

A popular approach is based on $2 \mathrm{D}$ slicing of point cloud where a set of points at a specified height is first selected and then trunks are found on the 2D sliced image by Hough transform or circle fittings (e.g., Maas, 2008 and Tansey, 2009). This approach enables efficient extraction of multiple trunks since data size of 2D image is much smaller than that of original point cloud and pixel connectivity of the image can be used for neighbourhood search in the extraction process. However this approach assumes that ground surface is flat, and it causes low versatility since it cannot be used for forest in the sloping land.

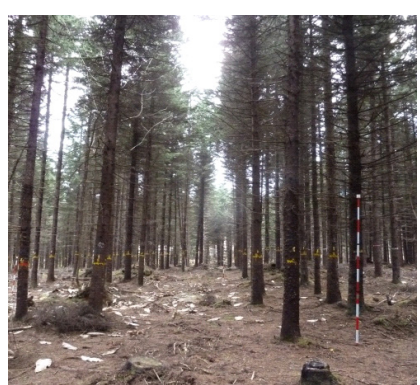

(a)

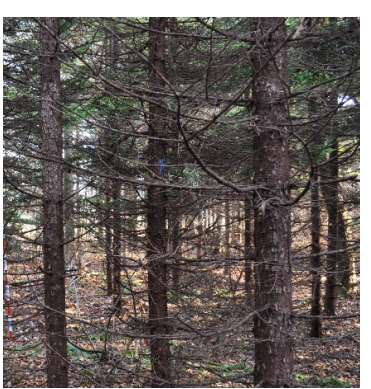

(b)

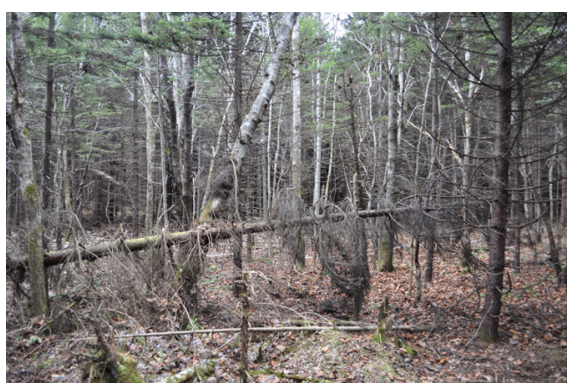

(c)

Fig.1 Our test site

Another approach is based on direct processing of 3D point cloud (Liang, 2012 and 2013). In this method, trunk points are detected by principal component analysis to a neighbourhood of each scanned point and then trunks are modelled by robust cylinder fittings to the points. This method enables extraction of multiple trunks with no assumption about ground surface. However it is reported that it fails detection when more than two trees are standing close to each other (Liang, 2012).

In summary, with existing automatic techniques, extraction rate is not sufficient in the case where trees are densely standing 


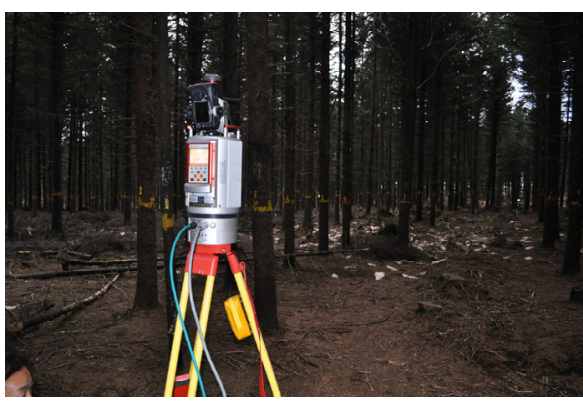

(a)

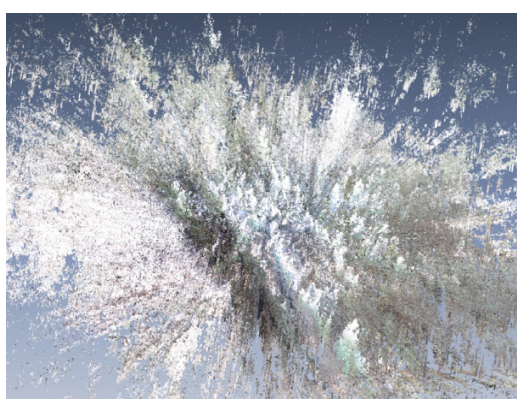

(b)

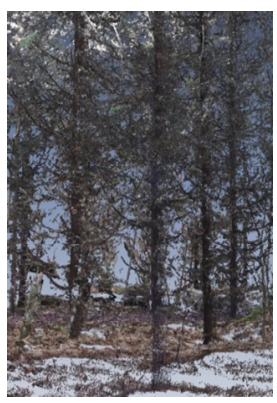

(c)
Fig.2 Laser-scanned point cloud of forest

Table 1 Specification of laser scanner

\begin{aligned} & \hline Field of view $360 \mathrm{deg} \times 100 \mathrm{deg} \\ &$\hline Measurement rate $122,000 \mathrm{point} / \mathrm{sec} \\ &$\hline Max measurement range $350 \mathrm{~m} \\ &$\hline Measurement accuracy $5 \mathrm{~mm}$ at $100 \mathrm{~m} \\ &$\hline Angular step $0.0024 \mathrm{deg} \times 0.0024 \mathrm{deg} \\ &$\hline Laser wavelength Near infrared \\ & \hline Laser beam divergence $0.35 \mathrm{mrad} \\ &$\hline\end{aligned}

and it becomes lower for trees farther from the scanning position. On the other hand, user-interactive techniques are successfully used in several applications of laser scans, such as as-built modeling of large facilities (Masuda, 2010). Although it should be practical to use user-interaction for trunk extraction from forest scans, such a technique has not been presented as far as authors know.

\subsection{Our purpose}

The goal of our work is to develop an interactive system for trunk extraction where a user can efficiently and intuitively detect trunks of all trees from forest point cloud captured by terrestrial laser scanner. In this paper, we present the basic system in which multiple trunks can be exhaustively extracted from a single scan and demonstrate its effectiveness. The main advantage of our interactive system over previous automatic techniques is the high extraction rate especially for trees which are densely standing each other and far from the scanning position.

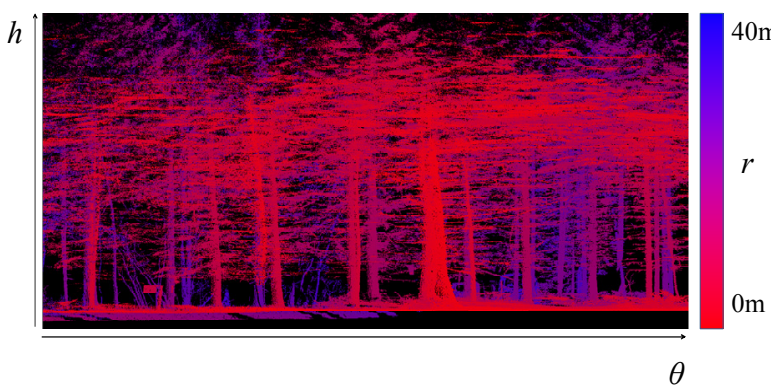

Fig.3 Distance image

\subsection{Test site and laser scanning}

In this work, we used the point cloud captured at picea glehnii forest in Tomakomai city in 2010 winter as shown in Fig.1(a). Here trees are densely standing and many branches exist, and several trees are fallen and leaned as shown in Fig.1(b) and (c), which make trunk extraction difficult. For the scanner, we used the RIEGL VZ-400 shown in Fig.2(a), which can capture point cloud with RGB color. Entire point cloud is shown in Fig.2(b) and a part of it is in Fig.2(c). Brief specification of the scanner is summarized in Table 1. For more details, please see the RIEGL website.

\section{OUR PROPOSED SYSTEM}

In this section, we describe an overview of our proposed system. Our system consist of two steps, automatic creation of distance image (step 1) and interactive trunk extraction (step 2). In the first step, given a single scan of forest, our system automatically creates a distance image from a point cloud centred at a scanning position. An example is shown in Fig.3. In the second step, as a user draws a line segment on the image so that it follows a target trunk, our system automatically and efficiently extracts a point subset which corresponds to a single trunk. Iterating the line drawings enables exhaustive extraction of multiple trunks separately.

It is possible to directly draw line segments on 3D point cloud with user-interactions. However in the visualization of point cloud, a view point is usually set outside the target (forest in our case) and visual recognition of internal tree trunks is difficult for a user. In contrast, our system set a view point inside a forest (a scanning position) on the image and this enables intuitive recognition and easy editing of trunks. We describe the details of each step in the following sections.

\subsection{Automatic creation of distance image (step 1)}

The laser scanner used in this work emits laser beams in the cylindrical way, e.g., 360deg in the horizontal and 100deg in the vertical directions respectively at a constant angle pitch. Therefore point cloud $\left\{x_{i}, y_{i}, z_{i}\right\}$ captured by a single scanning can be converted to the distance image centred at the scanning position using the following Eq.(1)-(3),

$$
r_{i}=\sqrt{x_{i}^{2}+y_{i}^{2}}
$$




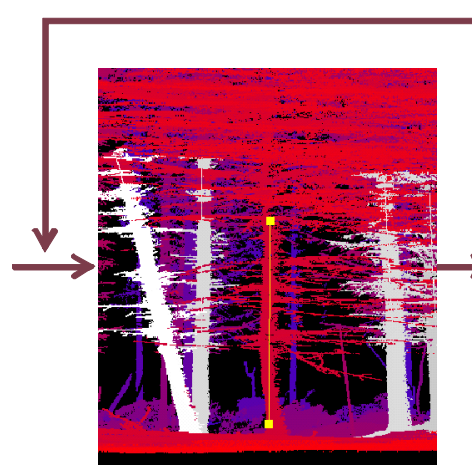

(a)

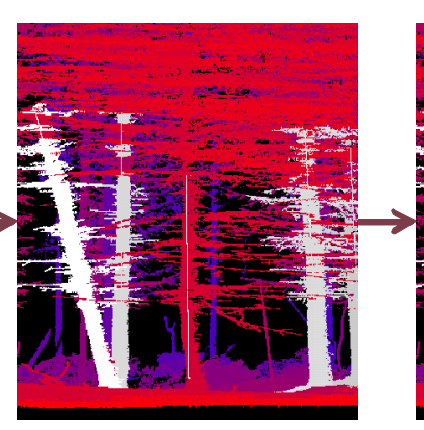

(b)

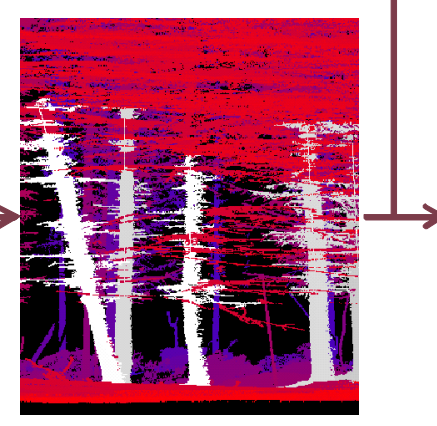

(c)

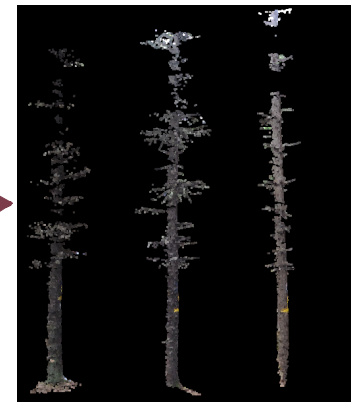

(d)

Fig.4 Overview of our interactive system

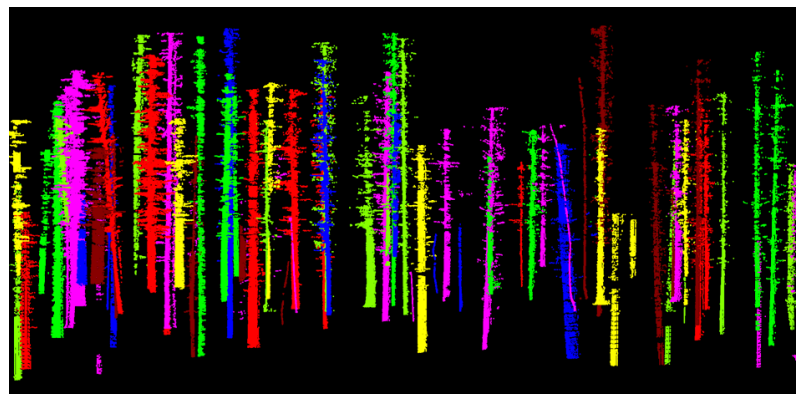

Fig.5 A part of extracted tree trunks by our system

$$
\begin{aligned}
& \theta_{i}=\left(\cos ^{-1} \frac{x_{i}}{r_{i}}\right) / \Delta \theta, \\
& h_{i}=z_{i} / \Delta h,
\end{aligned}
$$

where $r$ is the horizontal distance to the scanning position, $\theta$ is a horizontal angle, and $h$ is a height along the vertical upward direction. Since the angle pitch of the laser beam was $0.04 \mathrm{deg}$ in horizontal direction in our example, we set $\Delta \theta=0.04 \mathrm{deg}$. We also set $\Delta h=2 \mathrm{~cm}$ from several experiments.

\subsection{Interactive extraction of trunk (step 2)}

In our system, as a user draws a line segment on the distance image, then a system automatically extracts a point subset which corresponds to a single trunk. Details of each step is described below. An overview of our system is shown in Fig.4.

\section{Step 2-1: Interactive drawing of line segment}

Our current system require a user to select two pixels for drawing a line segment. A line segment is defined by connecting the two pixels. In this step, if a user selects a pixel on a high position of a target trunk in the image and the other on its low position as possible, then the system can robustly extracts corresponding point subsets of a target trunk from an entire point cloud. An example of this step is shown in Fig.4(a).

\section{Step 2-2: Automatic and efficient extraction of trunk points}

Next, our system automatically and efficiently extracts trunk points using the specified line segment by the region growing process as follows.

(1) A set of pixels $P=\left\{p_{j}\right\}$ crossing the line segment are
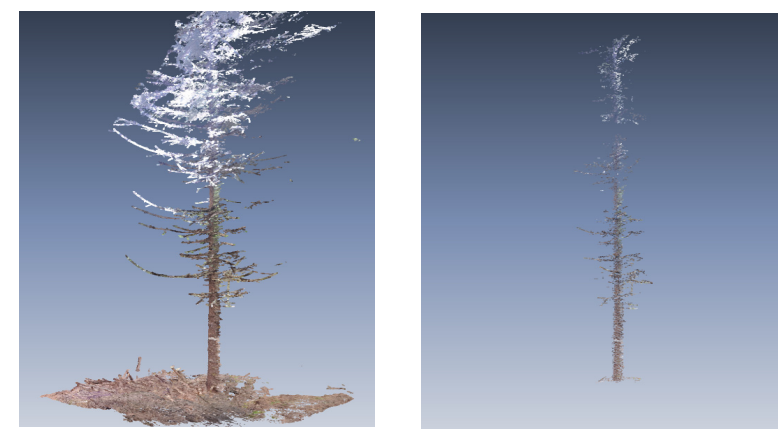

Fig.6 An example of extraction results of a single trunk (left: entire tree, right: tree trunk)

detected as shown in Fig.4(b) and their median distance $r_{\text {med }}$ of horizontal distance is computed.

(2) Allocate a unique label to the pixels among $P$ which satisfy the condition in Eq.(4).

$$
\left|r_{j}-r_{\text {med }}\right|<t h_{\text {med }}
$$

We set the threshold $t h_{\text {med }}$ to $30 \mathrm{~cm}$ for the examples in this paper because breast height diameters of our examples are ranging from $20 \mathrm{~cm}$ to $30 \mathrm{~cm}$.

(3) Neighboring pixels of the labeled pixels are recursively searched and similarly those satisfying the Eq.(4) are extracted and labeled. An example of the extracted pixels are shown in Fig.4(c).

(4) Finally, the corresponding points to the labeled pixels are extracted. An example is shown in Fig.4(d).

Iterating an interactive line drawing enables exhaustive extraction of multiple tree trunks. In the case of a coniferous tree, its trunk stretches toward upward vertical $(+z)$ direction and the horizontal distances of trunk points of a single tree are almost constant. However in the process of pixel selection on selected lines mentioned in section 2.1, several pixels may be extracted from areas other than target trunk, such as branches and leaves of front and back trees, and their horizontal distances much differ from those of the target trunk. Therefore we use median distance in the above process (2) so that target trunk points can be stably extracted. 


\section{RESULTS}

Fig. 5 and 6 show the extracted points by our system. In this example, 255 trunks were extracted. The number of trees in the point cloud was 299 which were visually counted. Thus the extraction ratio was $85.3 \%$ in total. As for the trees within $10 \mathrm{~m}$ from the scanning position, all of 16 trunks were correctly extracted, and for those from $10 \mathrm{~m}$ to $20 \mathrm{~m}, 23$ trunks of 25 were detected. Compared with the results by previous automatic techniques, our system achieved the extraction of far trees from the scanning position with high recognition rate (Liang, 2013). The main reason that several trunks cannot be extracted was the low point sampling at trees with many occlusions. Processing time for a single trunk extraction ranges from 3 to 5 seconds including line segment selection and image editing, such as translation and scaling, depending on the number of extracted points and visibility in the image. Total processing time was about 90 minutes including intermediate verifications of the results.

In our extraction results, several branches are detected with trunks and they must be deleted by post-processing using PCA analysis or circle-based RANSAC method automatically. In addition, several trunk points are remained not being extracted, especially at the top and bottom area of a trunk where a user cannot correctly select in interaction. Such points must be extracted also in the post-processing automatically.

\section{CONCLUSION AND FUTURE WORKS}

We proposed an interactive system for trunk extraction from a single laser scanned point cloud of forest. We demonstrated that our system could intuitively and efficiently detect most of the trunks. As for future works, we will extend our system to deal with multiple scans and use the extraction results for many forest applications, such as estimation of diameter at breast height or trunk volume. Usability test must be also carried out in order to verify operability and repeatability of our proposed system.

\section{REFFERENCE}

Hosoi, F. and Omasa, K., 2006. Voxel-Based 3-D Modeling of Individual Trees for Estimating Leaf Area Density Using HighResolution Portable Scanning Lidar. IEEE Transaction on Geoscience and Remote Sensing, 44(12), pp.3610-3618.

Kankare, V., Holopainen, M., Vastaranta, M., Puttonen, E., Yu, X., Hyyppä, J., Vaaja, M., Hyyppä, H. and Alho, P., 2013. Individual tree biomass estimation using terrestrial laser scanning. ISPRS Journal of Photogrammetry and Remote Sensing, 75, pp.64-75.

Kawashima, K., Kanai, S. and Date, H., 2014. As-built modeling of piping system from terrestrial laser-scanned point clouds using normal-based region growing. Journal of Computational Design and Engineering, 1(1), pp.13-26.

Lee, J., Son, H., Kim, C. and Kim, C., 2013. Skeleton-based 3D reconstruction of as-built pipelines from laser-scan data. Automation in Construction, 35, pp.199-207.
Liang, X., Litkey, P., Hyyppä, J., Kaartinen, H., Vastaranta, M. and Holopainen, M., 2012. Automatic Stem Mapping Using Single-Scan Terrestrial Laser Scanning. IEEE Transaction on Geoscience and Remote Sensing, 50(2), pp.661-670.

Liang, X. and Hyyppä, J., 2013. Automatic Stem Mapping by Merging Several terrestrial Laser Scans at the Feature and Decision Levels. Sensors, 13(2), pp.1614-1634.

Liang, X., Kankare, V., Yu, X., Hyyppä, J., and Holopainen, M., 2014. Automated Stem Curve Measurement Using Terrestrial Laser Scanning. IEEE Transaction on Geoscience and Remote Sensing, 52(3), pp.1739-1748.

Maas, H. G., Bienert, A., Scheller, S. and Keane, E., 2008. Automatic Forest Inventory Parameter Determination from Terrestrial Laser Scanner Data. International Journal of Remote Sensing, 29(5), pp.1579-1593.

Masuda, H. and Tanaka, I., 2010. As-Built 3D Modeling of Large Facilities Based on Interactive Feature Editing. Computer-Aided Design and Applications, 7(3), pp.349-360.

Mizoguchi, T., Koda, Y., Iwaki, I., Wakabayashi, H., Kobayashi, Y., Hara, Y., Shirai, K. and Lee, H. S., 2013. Quantitative scaling evaluation of concrete structures based on terrestrial laser scanning. Automation in Construction, 35, pp.263-274.

Molina, J. L., Rodríguez-Gonzálvez, P., Molina, M. C., González-Aguilera, D. and Espejo, F., 2014. Journal of Hydrology, 509, pp.150-162.

Raumonen, P., Kaasalainen, S., Kaasalainen, M. and Kaartinen, H., 2011. Approximation of Volume and Branch Size Distribution of Trees from Laser Scanner Data. International Archives of the Photogrammetry, Remote Sensing and Spatial Information Sciences, XXXVIII-5/W12, pp.79-84.

Tansey, K., Selmes, N., Anstee, A., Tate, N. J. and Denniss, A., 2009. Estimating Tree and Stand Variables in a Coesican Pine Woodland from Terrestrial Laser Scanner Data. International Journal of Remote Sensing, 30(19), pp.5195-5209.

Yu, X., Liang, K., Hyyppä, J., Kankare, V., Vastaranta, M. and Holopainen, M., 2013. Stem biomass estimation based on stem reconstruction from terrestrial laser scanning point clouds. Remote Sensing Letters, 4(4), pp.344-353.

Zheng, G. and Moskal, L. M., 2012. Leaf Orientation Retrieval From Terrestrial Laser Scanning (TLS) Data, 2012. IEEE Transaction on Geoscience and Remote Sensing, 50(10), pp.3970-3979.

\section{ACKNOWLEDGEMENT}

This work was financially supported by JSPS Grant-in-Aid for Young Scientists (B). We thank Shigeo Matsuda and Koichi Sasaki of RIEGL JAPAN for supporting the laser scanning. We also thank Keisuke Sato and Takuma Furihata for their coworking in this project. 